ACCEPTED TO ApJ, Preprint typset using LATEXstyle $_{\mathrm{E}}$

\title{
The Effect of Neutrino Radiation on Magnetorotational Instability in Proto-Neutron Stars
}

\author{
Youhei Masada $^{1,2}$, Takayoshi Sano ${ }^{3}$, and Kazunari Shibata ${ }^{1}$
}

\begin{abstract}
Neutrino radiation takes a major role in the momentum, heat, and lepton transports in protoneutron stars (PNSs). These diffusive processes affect the growth of magnetorotational instability (MRI) in PNSs. We perform a local linear analysis for the axisymmetric and nonaxisymmetric MRI including the effects of neutrino transports and ohmic dissipation. We find that the MRI can grow even in the multi-diffusive situations that are realized in neutrino loaded PNSs. When the toroidal magnetic component dominates over the poloidal one, nonaxisymmetric MRI modes grow much faster than axisymmetric modes. These results suggest the importance of the nonaxisymmetric MRI in PNSs. Thus the understandings of three-dimensional nonlinear evolutions of the MRI are necessary to reveal the explosion mechanism of core-collapse supernovae.
\end{abstract}

Subject headings: instabilities — MHD — stars : magnetic fields — stars : neutron

\section{INTRODUCTION}

Strong magnetic fields can play an important role on the dynamics of core-collapse supernovae. Akiyama et al. (2003) pointed out the possibility of the field amplification by the magnetorotational instability (MRI) in supernova core. The shapes of shock wave and the neutrinosphere are modified aspherically by the coupling effects of the rotation and magnetic fields, and anisotropic neutrino radiations could lead to jetlike explosions (Kotake et al.2003, 2004). Magnetic pressure driven explosions are also investigated in the context of magneto-rotational core collapse (Yamada \& Sawai 2004; Mizuno et al. 2004; Takiwaki et al. 2005; Ardeljan et al. 2005; Sawai et al. 2005; Moissenko et al. 2006). In these studies, strong magnetic fields ( $\gtrsim 10^{15} \mathrm{G}$ ) are generated by the field compression, field wrapping, and the MRI, which lead to the prompt explosions of core collapse supernovae. Alternative effects of magnetic fields are proposed by Thompson et al.(2005). They suggest that the turbulent viscosity sustained by the MRI converts the rotational energies of supernova core into the thermal energies, and thus the MRI will affect the dynamics of core collapse supernovae. However, these studies mainly focus on the dynamical effects of magnetic fields at the outside of PNSs. Therefore, magnetic phenomena inside PNSs and its influence on the core collapse dynamics have not been understood yet.

There are a few studies of magnetic effects at the interiors of PNSs. Miralles et al. (2002) show that the convective fluid motions in PNSs are prevented by strong magnetic fieds. Numerical simulations

\footnotetext{
${ }^{1}$ Kwasan and Hida Observatories, Kyoto University, Yamashina, Kyoto 607-8471, Japan; masada@kusastro.kyoto-u.ac.jp

${ }^{2}$ Department of Astronomy, Kyoto University, Sakyo, Kyoto 606-8502, Japan

${ }^{3}$ Institute of Laser Engineering, Osaka University, Suita, Osaka 565-0871, Japan
} 
suggest that the magnetic bubbles rise up by buoyancy and carry neutrino-rich materials to the neutron star surface (Wilson et al. 2005). This could increase the neutrino luminosity sufficietly to achieve a successful neutrino driven explosion. Socrates et al.(2005) show that the neutrino bubble instability grows at the inside of magnetized PNSs. Nonlinear evolution of the neutrino bubble instability will generate large density fluctuations. The radiation preferentially leaks out of the rarefied regions, which enhances the outgoing flux. They also propose that these global asymmetry in the neutino emission would cause the pulsar kicks.

Masada et al. (2006; hearafter Paper I) investigate the growth of the nonaxisymmetric MRI and show that it can grow even in the stably stratified envelope of PNSs. Our results suggest that the nonlinear evolution of MRI amplifies the magnetic fields and drives MHD turbulence in PNSs. The layers below the neutrinosphere are thought to be convectively stable. If buoyant motions are excited by the MRI, it could lead to enhancement of the neutrino luminosity. However, in Paper I, the effects of neutrino radiations on the growth of MRI are neglected. Below the neutrinosphere, electron-type neutrinos exchange energy with the matter via pair capture process and exchange momentum via elastic neutirno-nucleon scattering. These processes dominate the other transport processes by electrons and/or photons in PNSs, and thus the coefficients of the momentum, heat, and lepton transports are determined by neutrino radiative processes. The importance of these transports caused by the neutrino radiation have been suggested already in the previous studies of the mixing processes in PNSs (Bruenn \& Dinneva 1996; Mezzacappa et al. 1998; Miralles et al. 2000; Bruenn et al. 2004; Socrates et al. 2005). Therefore, we must study such neutrino effects to reveal the growth of MRI in more realistic, neutrino loaded PNSs.

In this paper, we analyze the diffusive effects caused by neutrino radiations on the growth of MRI and discuss the natures of MRI in PNSs. We consider four kinds of diffusive processes; the momentum, heat and chemical diffusions induced by the neutrino radiation and the ohmic dissipation of hot nuclear matters. As the mean free path of neutrino is short compared to the density and temperature length scales below the neutrinosphere, we can use diffusion approximation for the neutrino transports (Socrates et al. 2005). Another important feature of our analysis is to consider the vertical magnetic field as well as the toroidal one. For this case, both axisymmetric and nonaxisymmetric modes can be unstable for the MRI. In previous works, axisymmetric and nonaxisymmetric MRI are investigated separately (Acheson 1978; Balbus \& Hawley 1994; Menou et al. 2004). We consider both axisymmetric and nonaxisymmetric stability and discuss their difference in the growth rate and stability conditions.

The paper is organized as follows. In $\S 2$ we obtain the dispersion equation including diffusive processes, which determines the stability of magnetized PNSs. Necessary conditions for the stability are derived by an analytical approach. In $\S 3$ we discuss general properties of the dispersion relation focusing on the effects of quadruple diffusivities. In $\S 4$ we apply our results to the interior of magnetized PNSs, and examine the effects of neutrino radiations on the growth of MRI. We also examine the difference between axisymmetric and nonaxisymmetric modes of MRI. The possibility of the other instabilities is discussed in $\S 5$. Finally, we summarize our main findings in $\S 6$.

\section{LOCAL DISPERSION EQUATION}

\subsection{Physical assumptions and basic equations}

We examine the stability of differentially rotating, magnetized PNSs using a linear perturbation theory. In this paper, we focus on the effects of the neutrino radiation for MHD instabilities. The weak interaction between nuclear matters and neutrinos is described by using various opacities. We simply assume that 
the energy and momentum are exchanged between electron-type neutrinos and nuclear matters via pair capture process and elastic neutrino-nucleon scattering. In addition, neutrino opacities are assumed to be proportional to the square of the neutrino energy $E$,

$$
\kappa_{\nu}=\kappa_{0}\left(E / E_{0}\right)^{2},
$$

where $\kappa_{0}$ and $E_{0}$ are constants and independent of composition (e.g., Socrates et al.2005). In this paper, opacities are defined as the inverse of mean free paths.

Neutrinos are optically thick and both the thermal and chemical equilibrium would be held below the neutrinosphere. Thus, the governing equations are the following,

$$
\begin{gathered}
\frac{\partial \rho}{\partial t}+\nabla \cdot(\rho \boldsymbol{u})=0 \\
\frac{\partial \boldsymbol{u}}{\partial t}+(\boldsymbol{u} \cdot \nabla) \boldsymbol{u}=-\frac{1}{\rho} \nabla\left(P+\frac{1}{8 \pi} \boldsymbol{B}^{2}\right)+\frac{1}{4 \pi \rho}(\boldsymbol{B} \cdot \nabla) \boldsymbol{B}+\boldsymbol{g}+\nu \nabla^{2} \boldsymbol{u} \\
\frac{\partial \boldsymbol{B}}{\partial t}=\nabla \times(\boldsymbol{u} \times \boldsymbol{B})+\eta \nabla^{2} \boldsymbol{B} \\
n\left(\frac{\partial Y_{L}}{\partial t}+\boldsymbol{u} \cdot \nabla Y_{L}\right)=-\nabla \cdot \boldsymbol{F}_{L}, \\
n T\left(\frac{\partial s}{\partial t}+\boldsymbol{u} \cdot \nabla s\right)+n \mu_{\nu_{e}}\left(\frac{\partial Y_{L}}{\partial t}+\boldsymbol{u} \cdot \nabla Y_{L}\right)=-\nabla \cdot \boldsymbol{F} .
\end{gathered}
$$

These are the continuity equation, equation of motion, induction equation, conservations of lepton fraction and energy, respectively. Here $\rho$ is the fluid density, $\boldsymbol{u}$ is the flow velocity, $P$ is the total pressure of nuclear matters and radiations, $\boldsymbol{B}$ is the magnetic field, $\boldsymbol{g}$ is the gravitational field, $n$ is the baryon number density, $s$ is the entropy per baryon, $Y_{L}$ is the lepton fraction, $\mu_{\nu_{e}}$ is the neutrino chemical potential, and $\boldsymbol{F}$ and $\boldsymbol{F}_{L}$ are the radiative energy flux and lepton flux, respectively. Neutrino viscosity and magnetic diffusivity are represented by $\nu$ and $\eta$. The effect of the bulk viscosity is neglected here.

We employ the equilibrium diffusion approximation, and thus our analysis is restricted to the region below the neutrinosphere. Using the energy flux of the electron neutrino and anti-neutrino, $\boldsymbol{F}_{\nu_{e}}$ and $\boldsymbol{F}_{\overline{\nu_{e}}}$, the radiative energy flux $\boldsymbol{F}$ is given by

$$
\boldsymbol{F}=\boldsymbol{F}_{\nu_{e}}+\boldsymbol{F}_{\overline{\nu_{e}}}=-a_{T} \nabla T-a_{L} \nabla Y_{L},
$$

where

$$
a_{T}=\frac{E_{0}^{2}}{6 \kappa_{0} \hbar^{3} c^{2}} \frac{k_{B}^{2} T}{3}, \quad a_{L}=\frac{E_{0}^{2}}{6 \kappa_{0} \hbar^{3} c^{2}} \frac{\mu_{\nu_{e}}}{\pi^{2}}\left(\frac{\partial \mu_{\nu_{e}}}{\partial Y_{L}}\right)_{P, T}
$$

(Bludman \& Van Riper 1978). The lepton flux is given by

$$
\boldsymbol{F}_{L}=-b_{T} \nabla T-b_{L} \nabla Y_{L},
$$

where

$$
b_{L}=a_{L} / \mu_{\nu_{e}}=\frac{E_{0}^{2}}{6 \kappa_{0} \hbar^{3} c^{2}} \frac{1}{\pi^{2}}\left(\frac{\partial \mu_{\nu_{e}}}{\partial Y_{L}}\right)_{P, T} .
$$

Using the simple parameterization of opacity defined by equation (1), the temperature gradient cannot contribute to the lepton flux, that is $b_{T}=0$ (Socrates et al. 2005). Using equations (7) and (9), we can rewrite the conservations of lepton fraction and energy;

$$
n\left(\frac{\partial Y_{L}}{\partial t}+\boldsymbol{u} \cdot \nabla Y_{L}\right)=b_{L} \nabla^{2} Y_{L}
$$




$$
n T\left(\frac{\partial s}{\partial t}+\boldsymbol{u} \cdot \nabla s\right)=a_{T} \nabla^{2} T .
$$

We ignore the spatial dependence of the diffusion coefficients $a_{T}, a_{L}$, and $b_{L}$. This is appropriate for a local linear analysis. The resistive and viscous dissipation terms are also neglected in the energy equation, because they are higher order terms.

\subsection{Linearized Equations}

Following the analysis performed in Paper I, we consider the Eulerian perturbations (denoted by a prefix $\delta$ ) with the WKB spatial and temporal dependence, $\delta \propto \exp \left\{i\left(k_{r} r+m \phi+k_{z} z-\sigma t\right)\right\}$. Local WKB analysis is irrelevant in the cases that the growth rate of nonaxisymmetric unstable modes is smaller than the differential rotation rate. However, we now focus mainly on the MRI in the cases with strong velocity shear for which the growth rate is comparable to the angular velocity and adopt the simple WKB analysis in this paper. We use the cylindrical coordinates $(r, \phi, z)$ and consider a PNS rotating with the angular velocity $\Omega(r)$, and its magnetic fields being $\boldsymbol{B}=\left(0, B_{\phi}, B_{z}\right)$. We assume magnetic fields are uniform locally for simplicity. At the unperturbed state, the PNS is in the magnetohydrostatic equilibrium, that is,

$$
\frac{B_{\phi}^{2}}{4 \pi \rho r}+\frac{1}{\rho} \frac{\partial P}{\partial r}=-g_{r}+r \Omega^{2}, \quad \frac{1}{\rho} \frac{\partial P}{\partial z}=-g_{z},
$$

in the radial and vertical directions, respectively. Written out in component form and the largest terms retained, equations (2)-(4) become to linear order:

$$
\begin{gathered}
k_{r} \delta u_{r}+k_{z} \delta u_{z}=0, \\
i \omega_{\nu} \delta u_{r}+2 \Omega \delta u_{\phi}+\frac{i(\boldsymbol{k} \cdot \boldsymbol{B})}{4 \pi \rho} \delta b_{r}=\frac{i k_{r}}{\rho} \delta P+\frac{i k_{r}(\boldsymbol{B} \cdot \delta \boldsymbol{b})}{4 \pi \rho}+\frac{B_{\phi} \delta b_{\phi}}{2 \pi \rho r}-\frac{\delta \rho}{\rho}\left(g_{r}-r \Omega^{2}\right), \\
i \omega_{\nu} \delta u_{\phi}-\frac{1}{r} \frac{\partial\left(r^{2} \Omega\right)}{\partial r} \delta u_{r}+\frac{i(\boldsymbol{k} \cdot \boldsymbol{B})}{4 \pi \rho} \delta b_{\phi}+\frac{B_{\phi} \delta b_{r}}{4 \pi \rho r}=0, \\
i \omega_{\nu} \delta u_{z}+\frac{i(\boldsymbol{k} \cdot \boldsymbol{B})}{4 \pi \rho} \delta b_{z}=\frac{i k_{z}}{\rho} \delta P+\frac{i k_{z}(\boldsymbol{B} \cdot \delta \boldsymbol{b})}{4 \pi \rho}-\frac{\delta \rho}{\rho} g_{z}, \\
\omega_{\eta} \delta b_{r}+(\boldsymbol{k} \cdot \boldsymbol{B}) \delta u_{r}=0, \\
i \omega_{\eta} \delta b_{\phi}+i(\boldsymbol{k} \cdot \boldsymbol{B}) \delta u_{\phi}+r \frac{\partial \Omega}{\partial r} \delta b_{r}+\frac{B_{\phi}}{r} \delta u_{r}=0, \\
\omega_{\eta} \delta b_{z}+(\boldsymbol{k} \cdot \boldsymbol{B}) \delta u_{z}=0
\end{gathered}
$$

where

$$
\begin{gathered}
\omega=\sigma-m \Omega, \quad \omega_{\nu}=\omega+i \nu k^{2}, \quad \omega_{\eta}=\omega+i \eta k^{2}, \\
k^{2}=k_{r}^{2}+k_{z}^{2}, \quad k=\left(k_{r}, m / r, k_{z}\right), \quad \delta \boldsymbol{b}=\left(\delta b_{r}, \delta b_{\phi}, \delta b_{z}\right) .
\end{gathered}
$$

Here we use the local approximation $(k \gg 1 / r, m / r)$. We also adopt the Boussinesq approximation because the typical speeds considered in our analysis are much smaller than the sound speed. The relation $\delta P+$ $B_{\phi} \delta b_{\phi} / 4 \pi=0$ is used in equation (16) by virture of the local approximation (Acheson 1978).

To close linearized equations, we require another equation obtained from the thermodynamic processes. The chemical equilibrium is realized in PNSs, and thus the density is a function of pressure, temperature, and lepton fraction. In the Boussinesq approximation, pressure perturbations are negligible because fluid 
elements are assumed to be in the pressure equilibrium with their surroundings. Thus the density and entropy perturbations can be expressed in terms of the perturbations of temperature and lepton fraction:

$$
\begin{gathered}
\delta \rho=-\rho\left(\alpha \frac{\delta T}{T}+\beta \delta Y_{L}\right), \\
\delta s=m_{B} c_{p} \frac{\delta T}{T}+\zeta \delta Y_{L},
\end{gathered}
$$

where $m_{B}$ is the baryon mass, $\alpha=-(\partial \ln \rho / \partial \ln T)_{P, Y_{L}}, \beta=-\left(\partial \ln \rho / \partial Y_{L}\right)_{P, T}$ are the coefficients of thermal and chemical expansion, and $c_{p}=\left(T / m_{B}\right)(\partial s / \partial T)_{P, Y_{L}}$ is the specific heat at constant pressure. The sign of $\zeta=\left(\partial s / \partial Y_{L}\right)_{P, T}$ determines whether the leptonic gradient is stabilizing or destabilizing.

Equations (11) and (12) can be linearized and written in the form

$$
\begin{gathered}
-i \omega_{L} \delta Y_{L}+\delta \boldsymbol{u} \cdot \nabla Y_{L}=0 \\
-i \omega_{T} \delta T-\delta \boldsymbol{u} \cdot \nabla \Phi_{T}=\frac{\zeta T}{\rho c_{p}} b_{L} k^{2} \delta Y_{L}
\end{gathered}
$$

where

$$
\begin{gathered}
\omega_{T}=\omega+i \chi k^{2}, \omega_{L}=\omega+i \xi k^{2} \\
\nabla \Phi_{T}=-\frac{T}{m_{B} c_{p}}\left(\nabla s-\zeta \nabla Y_{L}\right)=\left(\frac{\partial T}{\partial P}\right)_{s, Y_{L}} \nabla P-\nabla T
\end{gathered}
$$

We introduce the heat diffusivity $\chi=a_{T} / \rho c_{p}$ and the chemical diffusivity $\xi=b_{L} / n$. Equation (26) represents the subadiabatic temperature gradient. Substituting equations (23) and (24) into equation (21), we obtain the linearized equation,

$$
i \omega_{T} \omega_{L} \frac{\delta \rho}{\rho}=\alpha \frac{\omega_{L}}{T}\left(\delta \boldsymbol{u} \cdot \nabla \Phi_{T}\right)-\beta\left[\omega+i(1+\psi) \chi k^{2}\right]\left(\delta \boldsymbol{u} \cdot \nabla Y_{L}\right)
$$

where

$$
\psi \equiv \frac{3}{\pi^{2}} \frac{\mu_{\nu_{e}}}{k_{B} T}\left(\frac{\partial \ln \mu_{\nu_{e}}}{\partial \ln T}\right)_{s, P}\left(\frac{\partial s / k_{B}}{\partial Y_{L}}\right)_{T, P} .
$$

Assuming the nondegenerate neutrino and anti-neutrinos in degenerate nuclear matters, $\psi$ is considered to be sufficiently smaller than unity (Burrows \& Lattimer 1986). Thus, equation (27) is rewritten as

$$
i \omega_{T} \omega_{L} \frac{\delta \rho}{\rho}=\alpha \frac{\omega_{L}}{T}\left(\delta \boldsymbol{u} \cdot \nabla \Phi_{T}\right)-\beta \omega_{T}\left(\delta \boldsymbol{u} \cdot \nabla Y_{L}\right)
$$

Eliminating perturbed quantities in eight linearized equations [eqs. (14) - (20) and (29)], we obtain the following dispersion equation:

$$
\begin{gathered}
\frac{k^{2}}{k_{z}^{2}} \omega_{D}^{4}-\frac{k^{2}}{k_{z}^{2}}\left[\frac{\omega_{\eta}}{\omega_{T}} N_{T e}^{2}+\frac{\omega_{\eta}}{\omega_{L}} N_{L e}^{2}\right] \omega_{D}^{2}-\kappa^{2} \omega_{R}^{2}\left[1+\frac{2 \omega_{A}^{2}}{\kappa^{2}} \frac{\omega_{D}^{2}}{\omega_{R}^{2}}\right] \\
-4 \Omega^{2}\left(\boldsymbol{k} \cdot \boldsymbol{v}_{\boldsymbol{A}}\right)^{2}\left[1+\left(\frac{\omega_{A}}{\Omega}\right)^{2}+\frac{4-q}{2}\left(\frac{\omega_{A}}{\Omega}\right)\left(\frac{\omega_{\eta}}{\boldsymbol{k} \cdot \boldsymbol{v}_{\boldsymbol{A}}}\right)+\frac{q}{2}\left(\frac{\omega_{A}}{\Omega}\right)\left(\frac{\omega_{\nu}}{\boldsymbol{k} \cdot \boldsymbol{v}_{\boldsymbol{A}}}\right)\right]=0
\end{gathered}
$$

where

$$
\begin{gathered}
\omega_{D}^{2}=\omega_{\nu} \omega_{\eta}-\left(\boldsymbol{k} \cdot \boldsymbol{v}_{\boldsymbol{A}}\right)^{2}, \quad \omega_{R}^{2}=\omega_{\eta}^{2}-\left(\boldsymbol{k} \cdot \boldsymbol{v}_{\boldsymbol{A}}\right)^{2} \\
\boldsymbol{v}_{\boldsymbol{A}}=\frac{\boldsymbol{B}}{\sqrt{4 \pi \rho}}=\left(0, V_{A \phi}, V_{A z}\right), \quad \kappa^{2}=\frac{1}{r^{3}} \frac{\partial}{\partial r}\left(r^{4} \Omega^{2}\right)
\end{gathered}
$$




$$
\begin{gathered}
\omega_{A}^{2}=\frac{V_{A \phi}^{2}}{r^{2}}=\frac{B_{\phi}^{2}}{4 \pi \rho r^{2}}, \quad \omega_{A z}^{2}=\frac{V_{A z}^{2}}{r^{2}}=\frac{B_{z}^{2}}{4 \pi \rho r^{2}}, \\
N_{T e}^{2}=-\frac{k_{z}^{2}}{k^{2}} \frac{\alpha}{\rho T}(D P) D \Phi_{T}, \quad N_{L e}^{2}=\frac{k_{z}^{2}}{k^{2}} \frac{\beta}{\rho}(D P) D Y_{L}, \\
D \equiv\left(\frac{\partial}{\partial r}-\frac{k_{r}}{k_{z}} \frac{\partial}{\partial z}\right), \quad q \equiv-\frac{d \ln \Omega}{d \ln r}
\end{gathered}
$$

Here, $\boldsymbol{v}_{\boldsymbol{A}}$ is the Alfven velocity, $\kappa$ is the epicyclic frequency, $\omega_{A}$ is the azimuthal Alfvén frequency, $\omega_{A z}$ is the vertical Alfvén frequency, $N_{T e}$ is the effective thermal buoyancy frequency, and $N_{L e}$ is the leptonic buoyancy frequency. The shear parameter $q$ denotes the rotational configuration of the systems.

Since the dispersion equation (30) has a very complex form, it is difficult to treat for what it is. Therefore, we simplify it using reasonable approximations. When we focus on the MRI modes with $\omega \sim \boldsymbol{k} \cdot \boldsymbol{v}_{\boldsymbol{A}}$ and adopt the weak magnetic field approximation $\Omega \gg \omega_{A}$, equation (30) can be described as

$$
\frac{k^{2}}{k_{z}^{2}} \omega_{D}^{4}-\frac{k^{2}}{k_{z}^{2}}\left[\frac{\omega_{\eta}}{\omega_{T}} N_{T e}^{2}+\frac{\omega_{\eta}}{\omega_{L}} N_{L e}^{2}\right] \omega_{D}^{2}-\kappa^{2} \omega_{R}^{2}-4\left(\boldsymbol{k} \cdot \boldsymbol{v}_{\boldsymbol{A}}\right)^{2} \Omega^{2}=0
$$

It is stressed that both axisymmetric and nonaxisymmetric MRI modes are included in this dispersion equation. In the diffusionless $(\nu=\eta=\chi=\xi=0)$ and pure toroidal $\left(B_{z}=0\right)$ limits, equation (31) is identical to the result of Paper I. Taking chemically homogenious $\left(\nabla Y_{L}=0\right)$ and axisymmetric $(m=0)$ limits, it reduces to the dispersion equation derived by Menou et al.2004.

\subsection{Stability Criterion}

The nature of instabilities in PNSs has been considered by a number of authors (Bruenn \& Dinneva 1996; Mezzacappa et al.1998; Miralles et al.2000,2002,2004). We make a detailed comparison of our dispersion equation with previous studies and derive the stability criterion for the MRI in the neutrino loaded PNSs.

Taking the diffusionless and hydrodynamic $(\boldsymbol{B}=0)$ limits, equation (31) is identical to the result of Miralles et al.2004, in which the stability conditions for the differentialy rotating PNSs are obtained. In the non-rotating limit $(\Omega=0$ ), equation (31) reduces to the results of Miralles et al.2002, in which they derive general stability criteria for the convective instability, taking dissipative processes into account such as neutrino transport, viscosity, and resistivity. Thus, the both semiconvective and neutron-finger unstable modes are obviously involved in our dispersion equation (Bruenn \& Dinneva 1996; Miralles et al.2000). It is, however, noticed that we neglect the influence of the chemical inhomogeneity on the heat diffusion and the thermal inhomogeneity on the chemical diffusion for simplicity.

Although the linear and nonlinear growth of semiconvection and neutron-finger instability would be a quite important issue in the evolution process of PNSs, it is beyond the scope of this paper. We now have an interest with the effects of multiple diffuions on the growth of MRI. Hence, we show the general stability criterion for the MRI in multi-diffusive systems, which is derived from equation (31) with the same procedure as in Urpin 2006. When we focus on relatively larger wavelengths $\left(\lambda \gg \lambda_{c} \equiv 2 \pi \sqrt{\nu \eta} / V_{A z}\right)$, the stability criterion for the MRI can be written as

$$
\frac{\eta}{\chi} N_{T}^{2}+\frac{\eta}{\xi} N_{L}^{2}+\frac{\mathrm{d} \Omega^{2}}{\mathrm{~d} \ln \mathrm{r}}>0
$$


where the thermal buoyancy frequency $N_{T}$ and the leptonic buoyancy frequency $N_{L}$ are defined by

$$
N_{T}^{2}=-\frac{\alpha}{\rho T}(\nabla P) \cdot \nabla \Psi_{T}, \quad N_{L}^{2}=\frac{\beta}{\rho}(\nabla P) \cdot \nabla Y_{L} .
$$

Equation (32) have a form similar to that derived in Urpin 2006 but with the leptonic gradient and the chemical diffusion. This criterion differs essentially from the standard stability criterion of the MRI (Balbus \& Hawley 1991,1994). Considering the diffusive coefficients which are typical in PNSs $(\chi, \xi, \gg \eta$ : see $\S 4.1)$, the stabilizing effect of the stratification is much reduced in our criterion. Here we notice that the viscous effect is not appeared in above criterion. However, the growth of MRI can be suppressed by the viscous damping (see $\S 3.2$ ). Thus we should consider not only the stability criterion but also the unstable growth rate when we discuss the characteristics of the MRI in multi-diffusive situations.

\section{GENERAL FEATURES OF THE DISPERSION EQUATION}

We solve the dispersion equation (31) numerically and examine the characteristics of MRI in multidiffusive situations. Note that, within the local approximation, the dispersion equation (31) is valid in the case $\Omega \gtrsim \boldsymbol{k} \cdot \boldsymbol{v}_{\boldsymbol{A}} \gg \omega_{A}$. As described above, both axisymmetic and nonaxisymmetic MRI modes are included in our dispersion equation. General features of MRI in such complicated situations are not fully investigated in previous studies. Our analysis could be applicable to not only the PNSs but also the other astrophysical objects, such as stellar interiors and accretion disks.

The effective thermal and leptonic buoyancy frequencies, $N_{T e}$ and $N_{L e}$, denote the size of the restoring force due to the stable stratification. Qualitative effect of the leptonic buoyancy is the same as thermal one. Thus we consider only the thermal buoyancy frequency as the measure of stabilizing effects due to the stratification for a while. In PNSs, the toroidal magnetic fields would dominate over the poloidal one. In the following, we fix the ratio of the toroidal to poloidal Alfvén frequencies as $\omega_{A} / \omega_{A z}=10^{4}$. The ratio of the angular velocity to the toroidal Alfvén frequency is assumed to be $\Omega / \omega_{A}=100$. These are about their typical values in rotating PNSs (see $\S 4$ ). Since we have an interest to the systems with differential rotation, the shear parameter $q=1.5$ is adopted in this section. Furthermore, we restrict our analysis to the modes with zero radial wavenumber $\left(k_{r}=0\right)$ which correspond to the fastest growing branch of the axisymmetric and nonaxisymmetric MRI.

\subsection{Diffusionless Limit}

We show the features of the dispersion relation in the diffusionless limit $(\nu=\eta=\chi=\xi=0)$. When the imaginary part of $\omega$ is positive, the amplitude of the perturbation will grow exponentially. Figure 1 shows a three-dimensional plot of the unstable growth rate as a function of the vertical and azimuthal wavenumbers. Stabilizing effects due to the stratification are ignored here $\left(N_{T e}=0\right)$. The growth rate is normalized by angular velocity $\Omega$. Both axisymmetric and nonaxisymmetric modes can be unstable for the MRI in this situation. For the case with $q=1.5$, the maximum growth rate of axisymmetric modes is $0.75 \Omega$ at $k_{z}=k_{\max }=0.97 \Omega / V_{A z}$. The cutoff wavenumber of axisymmetric modes depend on the strength of the vertical field, which is given by $k_{\text {crit }}=1.73 \Omega / V_{A z}$ (Balbus \& Hawley 1991). The maximum growth rate of nonaxisymmetric modes $(m \neq 0)$ is $0.75 \Omega$ at $m=m_{\max }=0.97 \Omega / \omega_{A}$. The cutoff wavenumber is determined by the strength of the toroidal field and given by $m_{\text {crit }}=1.73 \Omega / \omega_{A}$ (see Paper I). 
The stable stratification acts on the MRI as the negative buoyancy and reduces its growth rate. The amplitude of the restoring force is denoted by the size of $N_{T e}$. The stabilizing effect due to the stratification on the axisymmetric $(m=0)$ and nonaxisymmetric $\left(m=m_{\max }\right)$ MRI are depicted in Figure 2. The normalized growth rate is given by a function of the vertical wavenumber for the cases with different stabilizing parameters $N_{T e} / \Omega=0,0.5,1$ and 2 . The horizontal axes are measured by the logarithmic scale. As seen from these figures, the growth rate of MRI decreases as $N_{T e} / \Omega$ increases. When the stabilizing parameter exceeds a critical value $\left(N_{T e} / \Omega \gtrsim 2\right)$, the growth of MRI is completely suppressed. These results are generally approved in spite of the radial wavenumbers.

\section{2. $\quad$ Effects of Quadruple-Diffusions}

Our main interest in this paper is to understand the effects of multiple diffusions on the growth of MRI. In general, the viscous, heat, and chemical diffusions have a major impact on the growth of hydorodynamic and magnetohydrodynamic instabilities (Bruenn \& Dinneva 1996; Miralles et al. 2000, 2002; Bruenn et al. 2004). The ohmic dissipation could also affect the growth of MRI (Jin 1996; Sano \& Miyama 1999). We examine the single and multi-diffusive effects on the growth of MRI. We restrict our discussion to the case with zero radial wavenumber $\left(k_{r}=0\right)$. For further simplicity, we fix the azimuthal wavenumber as $m=m_{\max }$, which corresponds to the fastest growing mode of nonaxisymmetric MRI (see $\S 3.1$ ).

The dispersion relation is characterized by the normalized diffusivities defined as

$$
P e \equiv \frac{V_{A z}^{2}}{\chi \Omega}, \quad R e \equiv \frac{V_{A z}^{2}}{\nu \Omega}, \quad R e_{M} \equiv \frac{V_{A z}^{2}}{\eta \Omega},
$$

where $P e$ is the Peclet number, $R e$ is the Reynolds number, and $R e_{M}$ is the magnetic Reynolds number. Here, we select the vertical Alfvén speed as the typical velocity and $V_{A z} / \Omega$ as the typical lengthscale. The effect of the chemical diffusion is quite similar to that of the heat diffusion, and thus it is ignored for a while. We now consider the case with a strong toroidal field $\omega_{A} / \omega_{A z}=10^{4}$. The characteristic length scale of nonaxisymmetric MRI is thus much longer than that of axisymmetric MRI. Therefore, axisymmetric MRI is affected by the diffusion processes more severely than nonaxisymmetric MRI.

\subsubsection{Single Diffusive Effects}

It would be useful to describe the effects of each diffusions on the MRI separately. Figures 3a, 3b and $3 \mathrm{c}$ show the dispersion relation taking account of the heat, viscous, and magnetic diffusivities respectively. The horizontal axes of these figures are vertical wavenumber in the logarithmic scale.

Figure 3a demonstrates the effect of the heat diffusion. The other diffusive coefficients are assumed to be $\nu=\eta=0$. The heat diffusion can reduce the stabilizing effect due to the stratification and revive the unstable growth of MRI. As described in $\S 3.1$, the growth of MRI is suppressed by the restoring force of negative buoyancy. However, even with a strong stratification $\left(N_{T e} / \Omega \gtrsim 2\right)$, MRI can grow if the heat diffusion is efficient. This is because the heat diffusion induces the heat exchange between the fluid element and the surroundings, which eliminates the entropy difference between them. Thus it reduces the stabilizing effects caused by the entropy gradients and the growth of MRI is revived.

The typical wavelength $\lambda_{\chi}$ at which the heat diffusion can affect the buoyant oscillation is estimated 
from a simple relation, $\chi k^{2} \sim N_{T e}$, that is

$$
\lambda_{\chi}=2 \pi \sqrt{\frac{\chi}{N_{T e}}} .
$$

The heat diffusion affects the modes with shorter wavelengths than $\lambda_{\chi}$. On the other hand, the cutoff wavelength of MRI is given by $\lambda_{\mathrm{MRI}} \sim 2 \pi V_{A z} / \Omega$. Magnetic tensions stabilize the modes with longer wavelengths than $\lambda_{\text {MRI }}$. Thus we can derive the range of poloidal wavelength which involve fast growing MRI modes in the case with heat diffusion and stable stratification, that is $\lambda_{\mathrm{MRI}} \lesssim \lambda \lesssim \lambda_{\chi}$, in other words,

$$
1 \lesssim \bar{\lambda} \lesssim \bar{\lambda}_{\chi} \equiv \sqrt{\frac{\Omega}{N_{T e} P e}}
$$

where $\bar{\lambda} \equiv \lambda / \lambda_{\text {MRI }}$. Here we define the fast growing MRI modes as the modes whose growth rate is of the order of $q \Omega$. For the case with $N_{T e} / \Omega=2$, the condition for reviving the MRI is $P_{e} \lesssim 0.5$. This is consistent with the result shown in Figure 3a. In the strongly stratified regions $\left(N_{T e} / \Omega \gg 1\right)$, such as the outer layer of PNSs and the radiative core of the Sun, the condition $P_{e} \ll 1$ is needed for the growth of MRI.

Figure $3 \mathrm{~b}$ shows the viscous damping of the growth of MRI. Here $N_{T e}$ is assumed to be zero and the heat and magnetic diffusivities are neglected $(\chi=\eta=0)$. The effect of the kinetic viscosity is characterized by the Reynolds number Re. The Reynolds number is unity when the viscous damping rate is comparable to the growth rate of MRI, $\nu k^{2} \sim \Omega$. The growth of MRI is suppressed by the viscosity when $R e \lesssim 1$ as shown in Figure 3b. The critical wavelength is approximately given by

$$
\bar{\lambda}_{\nu} \equiv \frac{\lambda_{\nu}}{\lambda_{\mathrm{MRI}}}=\sqrt{\frac{\nu \Omega}{V_{A z}^{2}}}=\sqrt{\frac{1}{R e}},
$$

where $\lambda_{\nu} \equiv 2 \pi \sqrt{\nu / \Omega}$. We notice that the growth of MRI is suppressed but never stabilized by the viscous damping. This means that the stability condition of MRI is not affected by the viscous dissipation, which is discussed in $\S 2.3$. Thus we should consider both the growth rate and the stability condition when we discuss the characteristics of MRI in multi-diffusive systems.

Ohmic dissipative effects are shown in Figure 3c. The conditions $N_{T e}=0$ and $\chi=\nu=0$ are assumed in this figure. As seen from this figure, the effect of ohmic dissipation is similar to that of viscous dissipation. However, the ohmic dissipation can stabilize the MRI when the following condition is satisfied,

$$
\bar{\lambda} \lesssim \bar{\lambda}_{\eta} \equiv \frac{\lambda_{\eta}}{\lambda_{\mathrm{MRI}}}=\sqrt{\frac{1}{R e_{M}}}
$$

where $\lambda_{\eta} \equiv 2 \pi \sqrt{\eta / \Omega}$. Thus the stability condition of MRI depends on the magnetic diffusivity (see, $\S 2.3$ ). The ohmic dissipation becomes effective when $R e_{M} \lesssim 1$ is satisfied. This is also consistent with the Figure $3 \mathrm{c}$.

\subsubsection{Double Diffusive Effects}

Multi-diffusive processes often affect the stability of astrophysical objects. We investigate the double diffusive effect on the growth of MRI focusing on typical two cases. One is the double diffusive system with $\chi \gg \nu$ (PNS-type system), and the other is that with $\chi \gg \eta$ (SOLAR-type system). As described later, heat and viscous diffusions caused by the neutrino radiation dominate over the magnetic diffusion in PNSs 
$(\chi \gg \nu \gg \eta)$. On the other hand, the condition $\chi \gg \eta \gg \nu$ is satisfied in the solar radiative zone. We restrict our discussion to the cases with zero radial wavenumber and the azimuthal wavenumber $m=m_{\max }$.

Figure 4a shows the double diffusive effect on the growth of MRI in the PNS-type system. Vertical and horizontal axes are the same as Figure 3. The thick dashed-line shows the growth rate in the diffusionless and isentropic limits. The stabilization of MRI due to the stable stratification is depicted by the thick dotted-line $\left(N_{T e} / \Omega=2\right)$. The solid line shows the double diffusive effect of heat and viscous diffusions in the PNS-type system. We consider the case with $\chi \gg \nu$, so that $P e=10^{-4}$ and $R e=0.01$ are assumed here. Then the critical wavelengths are $\bar{\lambda}_{\chi}=10^{2}$ and $\bar{\lambda}_{\nu}=10$, respectively.

From this figure, it is found that the stabilizing effect is reduced and the growth of MRI is revived for the modes with the poloidal wavelengths shorter than $\lambda_{\chi}$. On the other hand, the unstable growth is suppressed by the viscous damping for the modes with the poloidal wavelengths shorter than $\lambda_{\nu}$. Therefore, in the PNS-type system, fast growing modes of MRI are limited to the following range;

$$
\max \left(\lambda_{\nu}, \lambda_{\mathrm{MRI}}\right) \lesssim \lambda \lesssim \lambda_{\chi}
$$

We show the relation between single and double diffusive cases. The thin dotted-line shows the single diffuisive case with the heat diffusion of $P e=10^{-4}$ and $N_{T e}=2.0$. The case with only the viscous diffusion of $R e=0.01$ and $N_{T e}=0$ is described by the thin dashed-line. We find that the double diffusive case is obviously superposed by each single diffusive cases.

Figure $4 \mathrm{~b}$ shows the double diffusive effect on the growth of MRI in the SOLAR-type system. Thick dashed and dotted-lines are the same as in Figure 4a. The solid line shows the double diffusive effect in the SOLAR-type system $(\chi \gg \eta)$. When we assume $P e=10^{-4}$ and $R e_{M}=0.01$, corresponding critical wavelengths are $\bar{\lambda}_{\chi}=10^{2}$ and $\bar{\lambda}_{\eta}=10$, respectively. As seen from this figure, the unstable growth of the MRI are revived by the effect of heat diffusion the same as in the PNS-type system. However, the ohmic dissipation stablize the unstable growth of MRI in the regime $\lambda \lesssim \lambda_{\eta}$. Therefore, in the SOLAR-type system, fast growing modes of MRI are limited to the following range,

$$
\max \left(\lambda_{\eta}, \lambda_{\mathrm{MRI}}\right) \lesssim \lambda \lesssim \lambda_{\chi}
$$

The SOLAR-type double diffusive case is also superposed by each single diffusive cases. The thin dashed-line shows the single diffuisive case with the thermal diffusion of $P e=10^{-4}$ and $N_{T e}=2.0$. The case with only the ohmic dissipation of $R e_{M}=0.01$ and $N_{T e}=0$ is depicted by the thin dotted-line. It is found that the condition of faster growing modes are determined by single diffusive behaviors of the disprsion relation.

Finally, we consider the general multi-diffusive systems $(\chi \neq 0, \xi \neq 0, \nu \neq 0, \eta \neq 0)$. It is indicated from the double diffusive systems that the lower limit of the wavelength for fast growing modes is determined by the two dissipation processes, that is the viscous and ohmic dissipation. In addition, its upper limit is determined by the two relaxation processes, that is the heat and lepton diffusions. Thus, in the multi-diffusive system, fast growing modes of MRI are limited to the following range:

$$
\max \left(\lambda_{\nu}, \lambda_{\eta}, \lambda_{\mathrm{MRI}}\right) \lesssim \lambda \lesssim \min \left(\lambda_{\chi}, \lambda_{\xi}\right)
$$

where $\lambda_{\xi} \equiv 2 \pi \sqrt{\xi / N_{L e}}$. In stably stratified regions $\left(N_{T e}, N_{L e}>\Omega\right)$ such as the envelope of PNSs and the solar radiative core, most of unstable MRI modes are drastically suppressed by the viscous damping, ohmic dissipation or negative buoyancy. Only the modes satisfying the condition given by equation (41) can grow significantly. The properties of the systems are determined by the fastest growing modes. It is, thus, important to know the typical lengthscale defined by the condition (41). 


\subsubsection{Difference in Axisymmetric and Nonaxisymmetric MRI}

We have investigated the diffusive effects on the nonaxisymmetric MRI. Generally, fast growing modes of axisymmetric MRI are also limited to the range defined by equation (41). However, the growth rate can differ among the axisymmetric and nonaxisymmetric MRI in multi-diffusive cases.

As seen from Figure 2, the growth rate of axisymmetric MRI decrease with increasing the poloidal wavelength, but that of nonaxisymmtric MRI remains to be constant even at $k_{z} \lesssim \Omega / V_{A z}$. Thus, in multidiffusive systems, the growth of nonaxisymmetric MRI dominates over that of axisymmetric one when the condition, $\max \left(\lambda_{\nu}, \lambda_{\eta}\right) \gg \lambda_{\mathrm{MRI}}$ is satisfied. In this case, fast growing modes are limited to the "window A" in Figures 2a and 2b. In the case satisfying the condition, $\max \left(\lambda_{\nu}, \lambda_{\eta}\right)<\lambda_{\text {MRI }}$, on the other hand, fast growing modes of axisymmetric and nonaxisymmetric MRI are limited to "window B" in Figures 2a and $2 \mathrm{~b}$, and their growth rates are comparable. Therefore, it is found that characters of fast growing modes of axisymmetric and nonaxisymmetric MRI are determined by the strength of vertical magnetic fields when the sizes of diffusive coefficients are fixed.

Note that these properties appear under the situation that typical wavelength of nonaxisymmetric MRI is much longer than that of axisymmetric one. The toroidal magnetic fields are generally considerd to be dominant over the poloidal one in the stellar context (Heger et al.2005). Therefore, these properties would be important in the envelope of PNSs and solar radiative core.

\section{APPLICATION TO PROTO-NEUTRON STARS}

In paper I, we have investigated the stability for the nonaxisymmetric MRI ignoring any diffusion processes and revealed that almost all regions of PNSs are unstable. However, we must consider the diffusive effects caused by the neutrino radiation in PNSs. The ohmic dissipation of hot nuclear matters can be also efficient in PNSs. Therefore, in this paper, the dispersion equation including multiple diffusion is derived. We apply it to the interiors of PNSs and reexamine the stability for the MRI. In the following, we restrict our discussion to the stably stratified regions of PNSs, which locates below the neutrinosphere (Janka \& Müller 1996; Thompson \& Murray 2000; Buras et al. 2003).

\subsection{Physical Quantities in Proto-Neutron Stars}

\subsubsection{Dynamical Quantities}

During the core collapse, a significant amount of differential rotation can be generated in PNSs, even if the precollapse core rotate rigidly (Heger et al.2000,2005). Numerical studies of the rotating core-collapse suggest that the envelope of PNSs would rotate at the angular velocity $\Omega \simeq 100-1000 \mathrm{sec}^{-1}$ with shear rate $q \lesssim 1$ (Buras et al.2003; Kotake et al.2003; Villain et al.2004). These values strongly depend on the precollapse core models. Observations of young isolated pulsars associated with supernova remnants indicate that they rotate with $\sim 100 \mathrm{sec}^{-1}$. Thus, we addopt $\Omega=100 \mathrm{sec}^{-1}$ and $q=1$ as typical rotational parameters.

From the stellar evolution calculations, we can estimate the strength of magnetic fields in PNSs. Heger et al. (2005) study the evolution of a magnetized massive star with rotation, and show that the precollapse iron core has spatially homogenious megnetic fields. Toroidal magnetic components are amplified from poloidal one via various dynamo processes in the stellar evolutionary phases (Spruit 1999, 2002). The toroidal 
component is about $\sim 10^{9} \mathrm{G}$, which dominates over the poloidal one $\sim 10^{6} \mathrm{G}$. Assuming the conservation of magnetic flux $\left(B \propto \rho^{2 / 3}\right)$, the envelope of the PNSs have $B_{\phi}=10^{13} \mathrm{G}$ and $B_{z}=10^{9} \mathrm{G}$. This would be the lower limit of the field strengths, because the magnetic fields could be amplified by turbulent dynamos or field wrapping during the core-collapse (Sawai et al.2005). Typical radius and density of PNSs are $R \sim 3 \times 10^{6} \mathrm{~cm}$ and $\rho \sim 10^{12} \mathrm{~g} \mathrm{~cm}^{-3}$. The corresponding Alfvén frequencies $\omega_{A}$ and $\omega_{A z}$ are given by

$$
\begin{aligned}
& \omega_{A}=1.0\left(\frac{B_{\phi}}{10^{13} \mathrm{G}}\right)\left(\frac{R}{3 \times 10^{6} \mathrm{~cm}}\right)^{-1}\left(\frac{\rho}{10^{12} \mathrm{~g} \mathrm{~cm}^{-3}}\right)^{-1 / 2} \mathrm{sec}^{-1}, \\
& \omega_{A z}=10^{-4}\left(\frac{B_{z}}{10^{9} \mathrm{G}}\right)\left(\frac{R}{3 \times 10^{6} \mathrm{~cm}}\right)^{-1}\left(\frac{\rho}{10^{12} \mathrm{~g} \mathrm{~cm}^{-3}}\right)^{-1 / 2} \mathrm{sec}^{-1} .
\end{aligned}
$$

The buoyancy frequencies in the stably stratified layer of PNSs are sensitive to the microscopic physics such as the equation of state and leptonic fraction (Buras et al.2003; Thompson et al.2005; Dessart et al.2005). In general, the sizes of the thermal and leptonic buoyancy frequencies are much larger than the angular velocity $\left(N_{T}^{2} \sim N_{L}^{2} \gg \Omega^{2}\right)$ and the both are of the same order below neutrinosphere (Janka \& Müller 1996). Thus, we assume that to be $N_{T} \sim N_{L}=10 \Omega$ in this section.

\subsubsection{Quadruple Diffusivities}

We estimate the various diffusion coefficients in PNSs. Under the equiriblium diffusion approximation, we can derive the heat and chemical diffusivities analytically as shown in $\S 2$. As the opacity $\kappa_{0}$, we adopt the simplified form derived by Janka (2001);

$$
\begin{aligned}
\kappa_{0} & \sim \frac{\rho \sigma_{0}}{m_{u}} \frac{\left\langle\epsilon_{\nu}^{2}\right\rangle}{\left(m_{e} c^{2}\right)^{2}} f\left(\alpha_{c}, Y_{n}, Y_{p}\right) \\
& =10^{-6}\left(\frac{\rho}{10^{12} \mathrm{~g} \mathrm{~cm}^{-3}}\right)\left(\frac{k_{B} T}{4 \mathrm{MeV}}\right)^{2}\left[\mathrm{~cm}^{-1}\right] .
\end{aligned}
$$

Here $\sigma_{0}=1.76 \times 10^{-44} \mathrm{~cm}^{2}$ is the characteristic weak interaction cross section, $m_{e} c^{2}=0.511 \mathrm{MeV}$ is the rest-mass energy of the electron, $m_{u}$ is the atomic mass unit, $f\left(\alpha_{c}, Y_{n}, Y_{p}\right) \approx 1$ is a function of the vector coupling constant $\alpha_{c}$, and the number fractions of free neutrons, $Y_{n}$, and protons, $Y_{p}$. The contributions from the neutral-current scattering and the charged-current absorption of the electron neutrino and the antielectron neutrino are included as the opacity source, but neutrino annihilation is not since the cross section is vanishingly small inside PNSs. Considering such the opacity, we can estimate the heat and chemical diffusivities as follows,

$$
\begin{aligned}
& \chi=10^{12}\left(\frac{\rho}{10^{12} \mathrm{~g} \mathrm{~cm}^{-3}}\right)^{-2}\left(\frac{E_{0}}{10 \mathrm{MeV}}\right)^{2}\left(\frac{k_{B} T}{4 \mathrm{MeV}}\right)^{-1}\left[\mathrm{~cm}^{2} \mathrm{sec}^{-1}\right] \\
& \xi=3 \times 10^{11}\left(\frac{\rho}{10^{12} \mathrm{~g} \mathrm{~cm}^{-3}}\right)^{-2}\left(\frac{E_{0}}{10 \mathrm{MeV}}\right)^{2}\left(\frac{k_{B} T}{4 \mathrm{MeV}}\right)^{-2}\left(\frac{\mu_{\nu_{e}}}{4 \mathrm{MeV}}\right)\left[\mathrm{cm}^{2} \mathrm{sec}^{-1}\right] .
\end{aligned}
$$

The viscous stress is induced by the neutrinos in a hot nuclear matter, and it acts on the larger scale compared to the neutrino mean free path. When we assume the nondegenerate electron neutrino and antielectron neutrino in degenerate nuclear matter, the heat diffusivity $\chi$ is related to the neutrino viscosity as

$$
\begin{aligned}
\nu & \sim \frac{c_{p} T}{\rho} \chi=m_{n}^{2 / 3} \rho^{-2 / 3} T^{2}\left[f\left(Y_{p}\right)\right]^{-1} \chi \\
& =10^{10}\left(\frac{\rho}{10^{12} \mathrm{~g} \mathrm{~cm}^{-3}}\right)^{-8 / 3}\left(\frac{E_{0}}{10 \mathrm{MeV}}\right)^{2}\left(\frac{k_{B} T}{4 \mathrm{MeV}}\right)\left[\mathrm{cm}^{2} \mathrm{sec}^{-1}\right]
\end{aligned}
$$


(van den Horn \& van Weert 1984; Thompson \& Duncan 1993), where $c_{p} \approx \rho^{1 / 3} m_{n}^{2 / 3} T\left[f\left(Y_{p}\right)\right]^{-1}$ is the specific heat at constant pressure per volume, $f\left(Y_{p}\right) \sim 1$ is a specific function of the protons number fraction (Thompson \& Duncan 1993). This is because both of the momentum and heat are transported by neutrinos dominantly.

The electric charge is transported via degenerate relativistic electrons there. Momentum transfer rate is mainly determined by electron-proton scattering in the case not exceeding the standard nuclear density $\sim 10^{14} \mathrm{~g} \mathrm{~cm}^{-3}$. When we consider the electron-proton collision, the electrical resistivity of hot nuclear matter is relatively low, $\mathcal{R}=6 \times 10^{-45} T^{2} \sec$ (Yakovlev \& Shalybkov 1991). Therefore, the magnetic diffusivity of the hot nucler matters is given by

$$
\eta=\frac{c^{2} \mathcal{R}}{4 \pi}=10^{-3}\left(\frac{k_{B} T}{4 \mathrm{MeV}}\right)^{2}\left[\mathrm{~cm}^{2} \mathrm{sec}^{-1}\right],
$$

The magnetic diffusivity is extremely smaller than the other diffusion coefficients in PNSs.

\subsection{Stability of Proto-Neutron Stars}

We discuss the characteristics of the axisymmetric and nonaxisymmetric MRI in the stably stratified regions of PNSs. In what follows, we use the physical quantities derived in $\S 4.1 ; q=1, \Omega=100 \mathrm{sec}^{-1}$, $\omega_{A}=1.0 \mathrm{sec}^{-1}, \omega_{A z}=10^{-4} \mathrm{sec}^{-1}$, and $N_{T}=N_{L}=10^{3} \mathrm{sec}^{-1}$. As the diffusivities, we addopt the values of $\chi=10^{12} \mathrm{~cm}^{2} \mathrm{sec}^{-1}, \xi=3 \times 10^{11} \mathrm{~cm}^{2} \mathrm{sec}^{-1}, \nu=10^{10} \mathrm{~cm}^{2} \mathrm{sec}^{-1}$, and $\eta=10^{-3} \mathrm{~cm}^{2} \mathrm{sec}^{-1}$. The features of MRI depend on the stellar merdian altitudes. Thus, we define the polar angle $\theta \equiv \tan ^{-1}(r / z)$ which denote the direction of the entropy and leptonic gradients. Using a polar angle $\theta$, the radial and vertical components of the buoyancy frequencies are written as $N_{r}=N \sin \theta$ and $N_{z}=N \cos \theta$.

\subsubsection{Stability of the equatorial region}

First of all, we discuss the growth of MRI at the equatorial plane of PNSs $(\theta=\pi / 2)$ in order to show the diffusive effects clearly. As described in Paper I, the equatorial region is stable to the MRI in the diffusionless case. This is because the stabilizing effect of the negative buoyancy becomes the maximum at the equatorial plane $\left(N_{T e}, N_{L e} \gg \Omega\right)$. Considering the diffusion processes, the stabilizing effect is reduced and the growth of MRI could be revived even at the equatorial regions.

Figure 5a shows the maximum growth rate of MRI $\left(k_{r}=0\right)$ at the equatorial plane of PNSs as a fuction of the azimuthal and vertical wavenumbers. The vertical wavenumber is normalized by the size of the system $R\left(=3 \times 10^{6} \mathrm{~cm}\right)$. In the Figure $5 \mathrm{~b}$, the slices of Figure $5 \mathrm{a}$ at $m=0$ and $m=m_{\max }$ are depicted. From these figures, we find that the maximum growth rate of nonaxisymmetric MRI $\left(\sim 10 \mathrm{sec}^{-1}\right)$ is significantly larger than that of axisymmetric one $\left(\sim 0.01 \mathrm{sec}^{-1}\right)$.

The range of poloidal wavelength which involve the fast growing modes of MRI is determined by the condition given by equation (41). Substituting the physical quantities, we obtatin the upper and lower bounds of the wavelength for the fast growing modes in PNSs as follows;

$$
10^{4} \lesssim \lambda \lesssim 10^{5} \quad[\mathrm{~cm}]
$$

This is surely consistent with the Figure 5 . The lower bound of this range is determined by the wavelength for the neutrino viscosity being effective. This means that the critical wavelength of axisymmetric MRI $\lambda_{\text {MRI }}$ 
is smaller than $\lambda_{\nu}$. In this case, fast growing modes of MRI are limited to the "window $A$ " in Figure 2. Therefore the growth of nonaxisymmetric MRI dominates over that of axisymmetric one (see $\S 3.2 .3$ ).

\subsubsection{Dependence of the maximum growth rate of MRI on stellar merdian altitudes}

The dependence of the maximum growth rate of MRI on stellar meridian altitudes $\theta$ is described in Figure 6. Upper and lower panels show the growth rate of axisymmetric and nonaxisymmetric MRI for the case with different field strengths $B_{z}=10^{9}, 10^{10}$, and $10^{12} \mathrm{G}$. As seen from these figures, the maximum growth rate decrease with increasing the polar angle $\theta$. This is because the stabilizing effect due to the stable stratification increases with polar angle $\theta$ (see Paper I). In addition, we can find that the maximum growth rate of axisymmetric MRI is strongly dependent on the strength of poloidal magnetic fields. When the strength of poloidal magnetic fields is weak $\left(\sim 10^{9-10} \mathrm{G}\right)$, nonaxisymmetric modes of MRI grow dominantly at any $\theta$. As the strength of poloidal fields increases, the maximum growth rate of axisymmetric MRI increases, and then becomes comparable to that of nonaxisymmetric MRI at $\sim 10^{12} \mathrm{G}$.

For the case with weak poloidal fields $\left(B_{z} \sim 10^{9-10} \mathrm{G}\right)$, the typical wavelength of axisymmetric MRI $\lambda_{\text {MRI }}$ is much smaller than $\lambda_{\nu}$. Thus the most growing mode of axisymmetric MRI is not included in the range defined by equation (49). This corresponds to the case that the fast growing modes are limited to the "window A" in Figure 2. Therefore, the nonaxisymmetric modes of MRI grow dominantly in this case. As the strength of poloidal fields increases, the length of $\lambda_{\text {MRI }}$ approachs to $\lambda_{\nu}$. This corresponds to the picture that the possible range of the fast growing modes shifts from "window $A$ " to "window B" in Figure 2. Thus the maximum growth rate of axisymmetric MRI increases when the strength of poloidal fields increases.

For the fast growth of axisymmetric MRI as much as that of nonaxisymmetric one, it is necessary to satisfy the condition $\lambda_{\nu} \lesssim \lambda_{\text {MRI }}$. Assuming the critical strength of poloidal fields satisfying this condition as $B_{c}$, we can derive the lower limit of poloidal fields for the fast growth of axisymmetric MRI;

$$
B \gtrsim B_{c} \sim(\rho \Omega \nu)^{1 / 2} \simeq 10^{12}\left(\frac{\rho}{10^{12} \mathrm{~g} \mathrm{~cm}^{-3}}\right)^{1 / 8}\left(\frac{\Omega}{100 \mathrm{sec}^{-1}}\right)^{1 / 2}\left(\frac{k_{B} T}{4 \mathrm{MeV}}\right)[\mathrm{G}] .
$$

Here we use the specific form of neutrino viscosity defined by equation (47). The growth of axisymmetric MRI is drastically suppressed by the neutrino viscosity when the strength of the poloidal magnetic fields is weaker than $\sim 10^{12} \mathrm{G}$. This critical value corresponds to that derived in Figure 5a numerically.

Note that, in the situation with realistic quadruple diffusions, the modes with zero radial wavenumber $\left(k_{r}=0\right)$ are the fastest growing modes. This is a remarkable difference from the diffusionless case that studied in Paper I, in which the modes with $k_{r} / k_{z}=N_{r} / N_{z}$ are most unstable one. That is, unstable fluid elements can move against the stratification in neutrino loaded PNSs. This character suggests that the MRI itself could operate as the process leading to overturnig fluid motions (Kato 1992; Balbus \& Hawley 1994).

As a result, we conclude that stably stratified envelope of PNSs are always unstable to the MRI. Especially, the maximum growth rate of nonaxisymmetric MRI is significantly larger than that of axisymmetric MRI unless the poloidal magnetic components are extremely strong $\left(\gtrsim 10^{12} \mathrm{G}\right)$. Typical growth time of MRI is about $100 \mathrm{msec}$. This is much shorter than the neutrino cooling time of PNSs ( $\sim 10 \mathrm{sec})$, and thus, it is expected that the nonlinear growth of MRI affect the neutrino luminosity via effective heating processes caused by overturnig fluid motions and/or the other magnetic processes. 


\section{DISCUSSION}

The nonlinear evolution of the MRI could amplify the magnetic fields and drive MHD turbulence in PNSs. On the other hand, the growth of other instabilities is also expected (Bruenn \& Dinneva 1996; Miralles et al. 2000, 2002; Bruenn et al. 2004). In particular, the radiative-driven magnetoacoustic instability, which is refered as "neutrino bubble", is focused as the seed process of the turbulent mixing and the mechanism for generating pulsar kicks (Blaes \& Socrates 2003; Socrates et al. 2005). We compare the growth rates of the MRI and the neutrino bubble instability, and derive the condition for the MRI dominating over the neutrino bubble instability.

The growth rate of neutrino bubble instability is given by

$$
\gamma_{\max } \simeq 3 \times 10^{2}\left(\frac{B_{p}}{10^{15} \mathrm{G}}\right)\left(\frac{g}{10^{13} \mathrm{~cm} \mathrm{sec}^{-2}}\right)\left(\frac{T}{4 \mathrm{MeV}}\right)^{-1}\left(\frac{\rho}{10^{12} \mathrm{~g} \mathrm{~cm}^{-3}}\right)^{-1 / 2} \mathrm{sec}^{-1},
$$

where $B_{p}$ is poloidal magnetic fields (Socrates et al. 2005). Considering the typical growth rate of MRI $(\sim q \Omega)$, we can derive the condition for the MRI dominating the neutrino bubble instability at the certain situation of $T \sim 4 \mathrm{MeV}, \rho \sim 10^{12} \mathrm{~g} \mathrm{~cm}^{-3}$, and $g \sim 10^{13} \mathrm{~cm} \mathrm{sec}^{-2}$ :

$$
\Omega \gtrsim 3 \times 10^{-7} q^{-1} B_{p}
$$

This condition suggests that the MRI dominates over the neutrino bubble instability in the weakly magnetized systems with a rapid differential rotaion.

From the condition (52), we can speculate the evolutionary scenario of PNSs. As PNSs should rotate differentially and be magnetized weakly right after core bounce, the growth of MRI exceeds that of the neutrino bubble instability. In the early evolutionaly phase, the nonlinear evolution of MRI initiates and sustains MHD turbulence. Thus, turbulent motions induced by MRI would enhance the neutrino luminosity sufficiently to yield explosions. MHD turbulence leads to angular momentum transport. Thus, the rotational configurations within PNSs evolve toward a rigid rotation. If the PNSs rotate rigidly, the neutrinosphere becomes oblate, and the neutrino luminosity could be enhanced in the polar region (Kotake et al.2003,2004). Thus, the polar region would be heated by neutrinos preferentially.

After magnetic fields are amplified by the MRI and the rotation profile of PNSs becomes the rigid rotation, the growth rate of the neutrino bubble instability can be comparable to that of the MRI. At this evolutionary phase, the neutrino bubble instability can grow nonlinearly and drive turbulent mixing as is suggested in Socrates et al.(2005). Local luminosity enhancements, which preferentially occur in the regions of strong magnetic field, lead to a net global asymmetry in the neutrino emission and the young neutron star is propelled in the direction opposite to these regions. In addtion, the magnetic buoyancy instability may also grow in PNSs (Masada et al. 2006 in prep). To reveal the origin of neutron star kicks completely, we should study the nonlinear coupling effects of these instabilities in detail.

\section{SUMMARY}

In this paper, we investigate the stabiliy of differentially rotating, magnetized PNSs, including the effects of multiple-diffusions. We derive the local dispersion equation for both axisymmetric and nonaxisymmetric perturbations and apply it to the interior of PNSs. Our findings are summarized as follows.

1. The heat and leptonic diffusions reduce the restoring force due to stable stratification and increase the maximum growth rate. The viscous damping suppresses the growth of short wavelength perturbations 
and decrease the maximum growth rate. The ohmic dissipation stabilizes short wavelength perturbations. In the multi-diffusive systems, the fast growing modes of MRI can be seen only when the poloidal wavelength is

$$
\max \left(\lambda_{\nu}, \lambda_{\eta}, \lambda_{\mathrm{MRI}}\right) \lesssim \lambda \lesssim \min \left(\lambda_{\chi}, \lambda_{\xi}\right)
$$

The upper bound is determined by the lengthscale that the restoring force due to the stable stratification is reduced by the heat or leptonic diffusion. The lower bound is determined by the lengthscale for ohmic dissipation or viscous damping being effective.

2. The necessary conditions of the stability for MRI in multi-diffusive case are derived from our simplified dispersion equation. The conditions are similar to that of Menou et al.(2004), in which only the stability for axisymmetric MRI is investigated.

3. MRI can grow in realistic neutrino loaded PNSs. For the case with $B_{\phi} \gg B_{z}$, the growth of nonaxisymmetric MRI dominates over axisymmetric one unless the vertical field is extremely strong $\left(B_{z} \gtrsim\right.$ $10^{12} \mathrm{G}$ ). Typical growth time of fast growing MRI modes is about $100 \mathrm{msec}$, which is much shorter than the neutrino cooling time of PNSs.

4. The condition that the growth of the MRI dominates over the neutrino bubble instability is given by

$$
\Omega \gtrsim 3 \times 10^{-7} q^{-1} B_{p}
$$

at $T \sim 4 \mathrm{MeV}, \rho \sim 10^{12} \mathrm{~g} \mathrm{~cm}^{-3}$ and $g \sim 10^{13} \mathrm{~cm} \mathrm{sec}^{-2}$. Right after the core bounce, the growth of the MRI is dominant over the neutrino bubble instability. After the strong magnetic field is generated by the MRI and the rotational configuration shift to a rigid shape, the growth rate of the neutrino bubble instability could become larger than that of the MRI.

We thank V. Urpin and S. Nagataki for helpful discussions. TS is supported by the Grant-in-Aid (16740111, 17039005) from the Ministry of Education, Culture, Sports, Science, and Technology of Japan.

\section{REFERENCES}

Acheson, D. J. 1978, Phil. Trans. Roy. Soc. Lond. A 289, 459

Akiyama, S., Wheeler, J. C., Meier, D. L., \& Lichtenstadt, I. 2003, ApJ, 584, 954

Ardeljan, N. V., Bisnovatyi-Kogan, G. S., Moiseenko, S. G. 2005, MNRAS, 359, 333

Balbus, S. A., \& Hawley, J. F. 1991, ApJ, 376, 214

Balbus, S. A., \& Hawley, J. F. 1994, MNRAS, 266, 769

Balbus, S. A. 2001, ApJ, 562, 909

Blaes, O., \& Socrates, A. 2003, ApJ, 596, 509

Bludman, S. A., \& van Riper, K. A. 1978, ApJ, 224, 631

Bruenn, S. W., \& Dineva, T. 1996, ApJ, 458, L71

Bruenn, S. W., Raley, E. A., \& Mezzacappa, A. 2004, preprint (astro-ph/0404099) 
Buras, R., Rampp, M., Janka, H.-T., \& Kifonidis, K. 2003, Phys. Rev. Lett., 90, 241101

Burrows, A., \& Lattimer, J. 1986, ApJ, 307, 178

Dessart, L., Burrows, A., Livne, E., \& Ott, C. D. 2005, preprint(astro-ph/0510229)

Goldreich, P., \& Schubert, G. 1967, ApJ, 150, 571

Heger, A., Langer, N., \& Woosley, S. E. 2000, ApJ, 528, 368

Heger, A., Woosley, S. E., Langer, N., \& Spruit, H. C. 2005, ApJ, 626, 350

Janka, H.-T., \& Müller, E. 1996, A\&A, 306, 167

Janka, H.-T. 2001, A\&A, 368, 527

Jin, L. 1996, ApJ, 457, 798

Kato, S. 1992, PASJ, 44, L31

Keil, W., Janka, H.-T., \& Müller, E. 1996, ApJ, 473, L111

Kotake, K., Yamada, S., \& Sato, K. 2003, ApJ, 595, 304

Kotake, K., Sawai, H., Yamada, S., \& Sato, K. 2004, ApJ, 608, 391

Masada, Y., Sano, T., \& Takabe, H. 2006, ApJ, 641, 447

Menou, K., Balbus, S. A., \& Spruit, H. C. 2004, ApJ, 607, 564

Mezzacappa, A., Calder, A. C., Bruenn, S. W., Blondin, J. M., Guidry, M. W., Strayer, M. R., \& Umar, A. S. 1998, ApJ, 493, 848

Miralles, J. A., Pons, J. A., \& Urpin, V. A. 2000, ApJ, 543, 1001

Miralles, J. A., Pons, J. A., \& Urpin, V. A. 2002, ApJ, 574, 356

Miralles, J. A., Pons, J. A., \& Urpin, V. 2004, A\&A, 420, 245

Mizuno, Y., Yamada, S., Koide, S. \& Shibata, K. 2004, ApJ, 615, 389

Moiseenko, S. G., Bisnovatyi-Kogan, G. S., \& Ardeljan, N. V. 2006, preprint(astro-ph/0603789)

Sano, T., \& Miyama, S. M. 1999, ApJ, 515, 776

Sawai, H., Kotake, K., \& Yamada, S. 2005, ApJ, 631, 446

Socrates, A., Blaes, O., Hungerford, A., \& Fryer, C. L. 2005, ApJ, 632, 531

Spruit, H. C. 1999, A\&A, 349, 189

Spruit, H. C. 2002, A\&A, 381, 923

Takiwaki, T., Kotake, K., Nagataki, S., \& Sato, K. 2004, ApJ, 616, 1086

Thompson, C., \& Duncan, R. C. 1993, ApJ, 408, 194 
Thompson, C., \& Murray, N. 2000, ApJ, 560, 339

Thompson, T. A., Quataert, E., \& Burrows, A. 2005, ApJ, 620, 861

Urpin, V. 2006, A\&A, 447, 285

van den Horn, L. J., \& van Weert, C. G. 1984, A\&A, 136, 74

Villain, L., Pons, J. A., Cerdá-Durán, P., \& Gourgoulhon, E. 2004, A\&A, 418, 283

Wilson, J. R., Mathews, G. J., \& Dalhed, H. E. 2005, ApJ, 628, 335

Yakovlev, D. G., \& Shalybkov, D. A. 1991, Ap\&SS, 176, 191

Yamada, S., \& Sawai, H. 2004, ApJ, 608, 907 


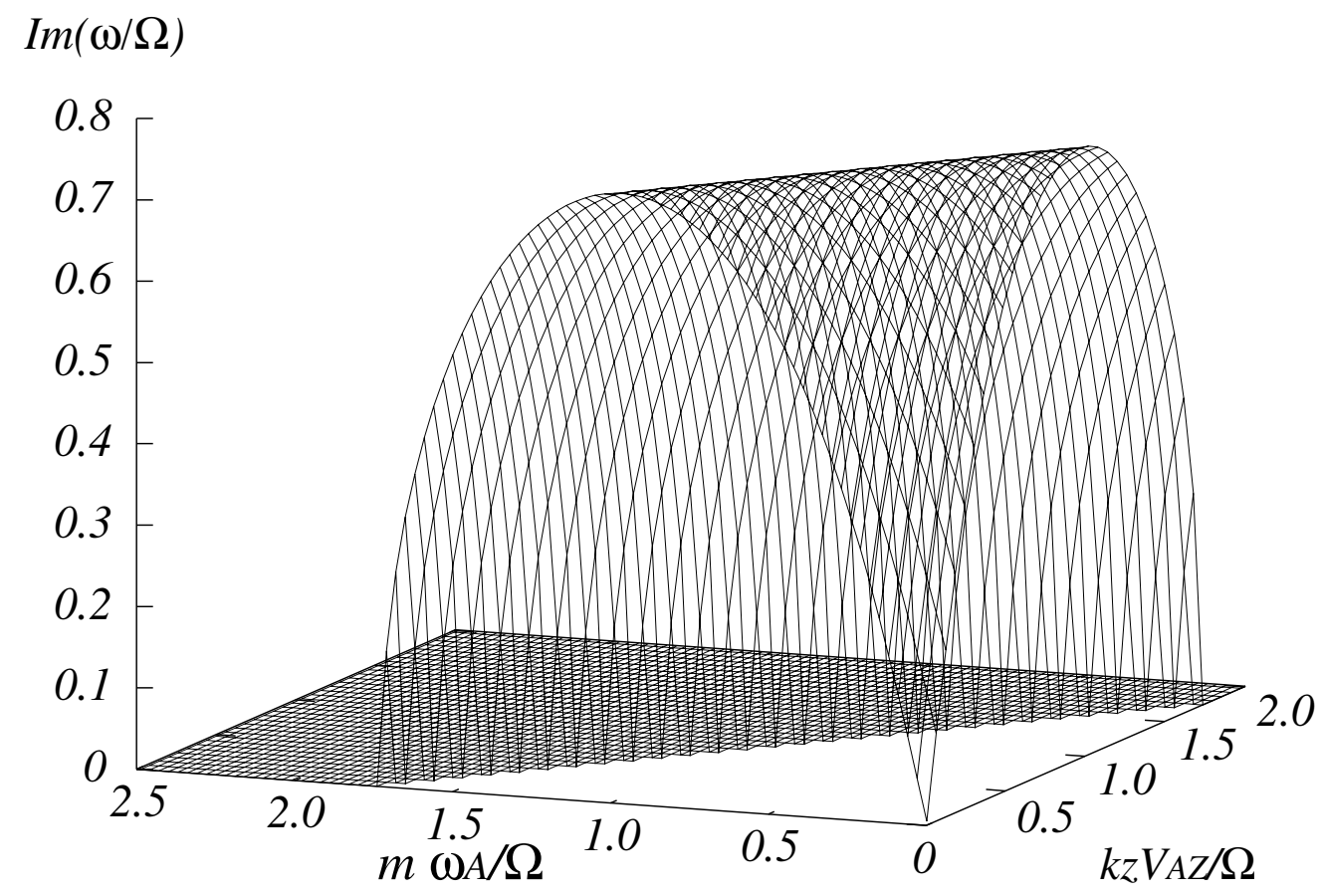

Fig. 1. - Unstable growth rate $\Im(\omega)$ in the diffusionless case is plotted as a function the azimuthal wavenumber $m$ and the vertical wavenumber $k_{z}$. For simplicity, we select the case of zero radial wavenumber $k_{r}=0$. The growth rate is normalized by the angular velocity $\Omega$, and the wavenumbers are normalized by $\omega_{A} / \Omega$ and $V_{A z} / \Omega$, respectively. In this figure, we assume the shear parameter $q=1.5$, the effective thermal and leptonic buoyancy frequencies $N_{T e}=N_{L e}=0$. Quadruple diffusivities are assumed to be zero $(\chi=\xi=\nu=\eta=0)$. 

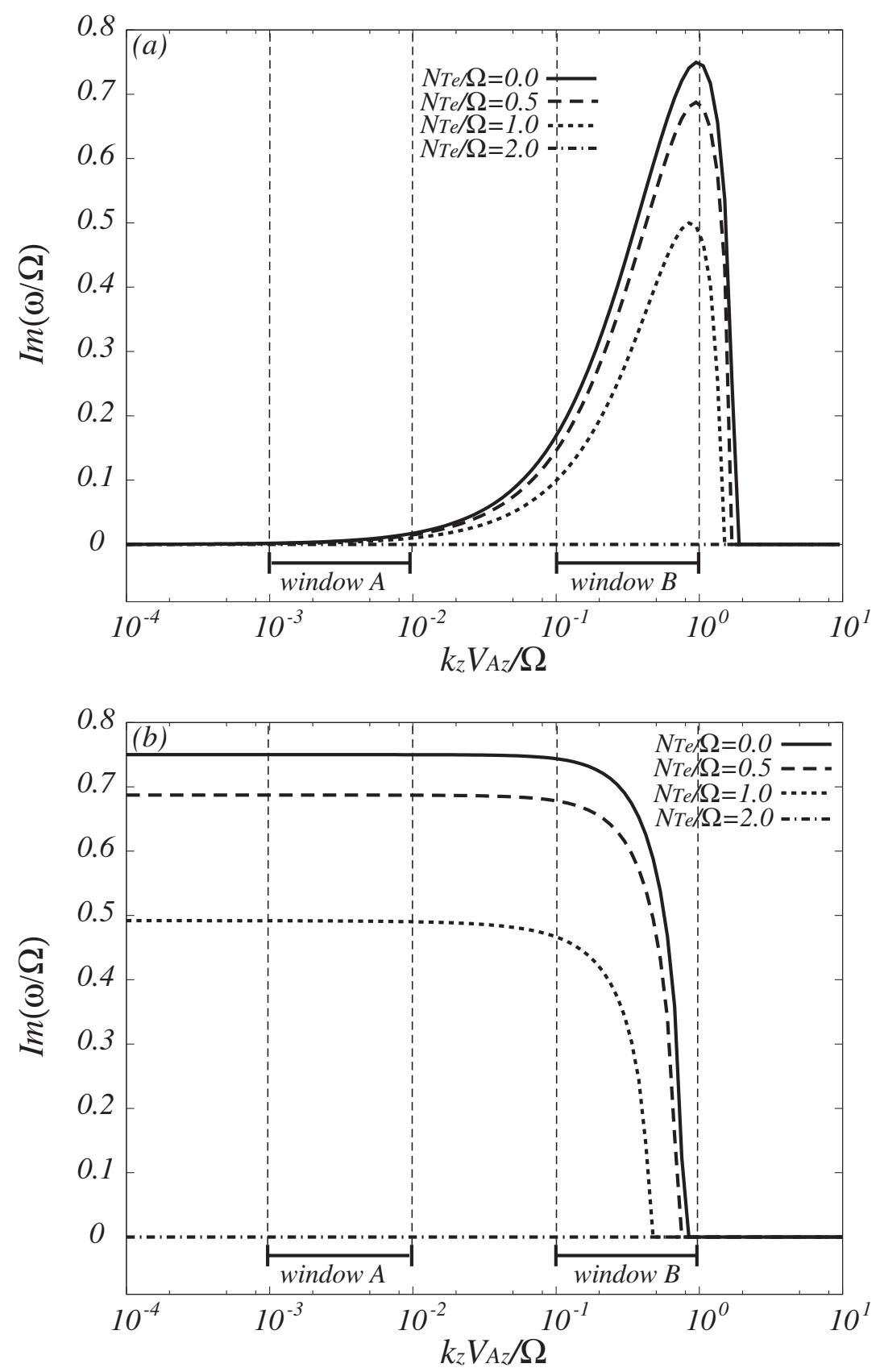

Fig. 2.- Dependence of the growth rate on the stabilizing parameter $N_{T e} / \Omega$ for the case of (a) axisymmetric MRI $(m=0)$, and (b) nonaxisymmetric MRI $\left(m=m_{\max }\right)$. The growth rate is shown as a function of the normalized vertical wavenumber $k_{z} V_{A z} / \Omega$ for the case $N_{T e} / \Omega=0,0.5,1$, and 2 , respectively. Note that the horizontal axis is measured by the logarithmic scale. Model parameters are the same as those in Figure 1. The growth rate of axisymmetric and nonaxisymmetric MRI decrease as the stabilizing parameter increases. When $N_{T e} \gtrsim 2 \Omega$, the growth of the MRI is completely stabilized by the stable stratification. 

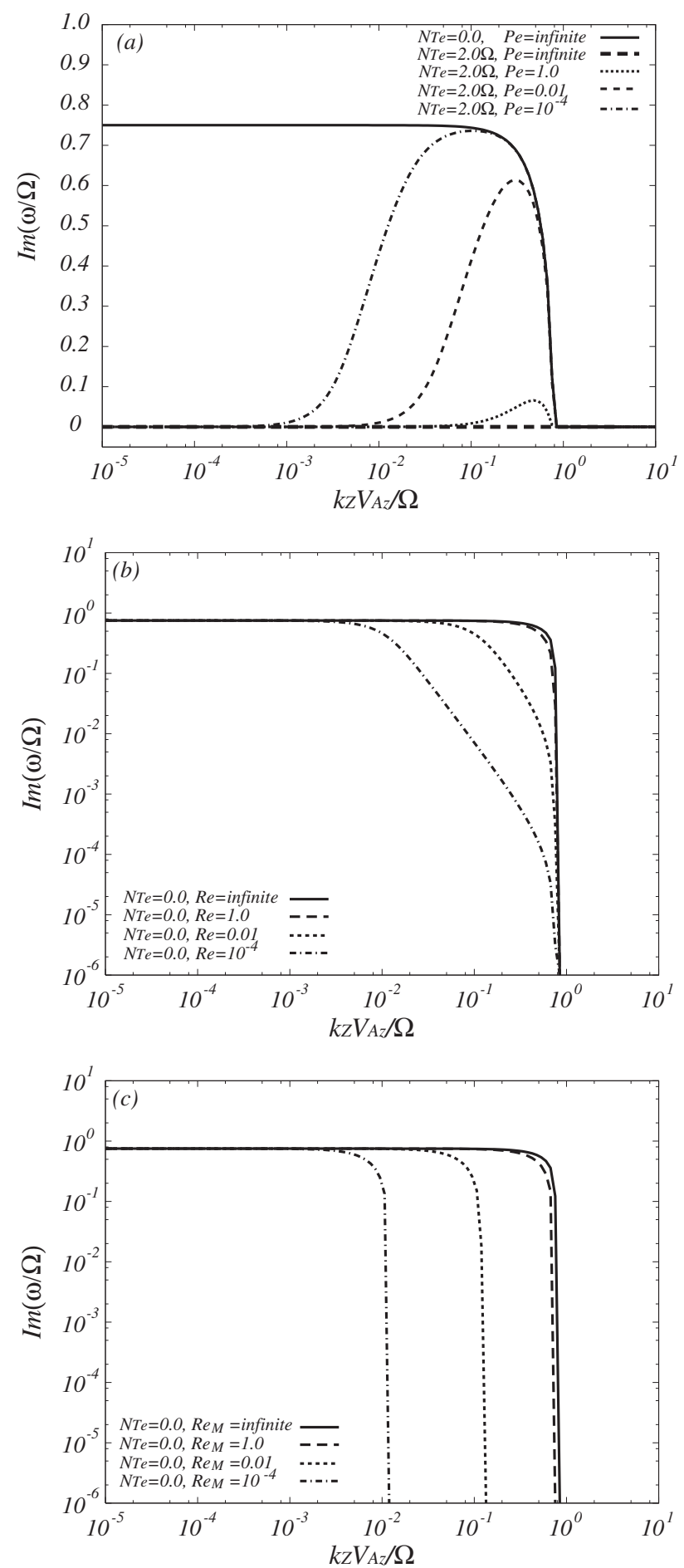

Fig. 3.- Single diffusive effects of (a) heat, (b) viscous, and (c) magnetic diffusions on the growth of MRI. Normalized growth rates are shown as a function of the normalized vertical wavenumber $k_{z} V_{A z} / \Omega$. We use the Peclet number $P e$, the Reynolds number $R e$ and the magnetic Reynolds number $R e_{M}$ as the measures of the effects of heat, viscous, and mangetic diffusivities respectively. For simplicity, we select the branch with $m=m_{\max }$ and $k_{r}=0$. This corresponds to the fastest growing branch of the nonaxisymmetric MRI. The other parameters are the same as in Figure 1. 

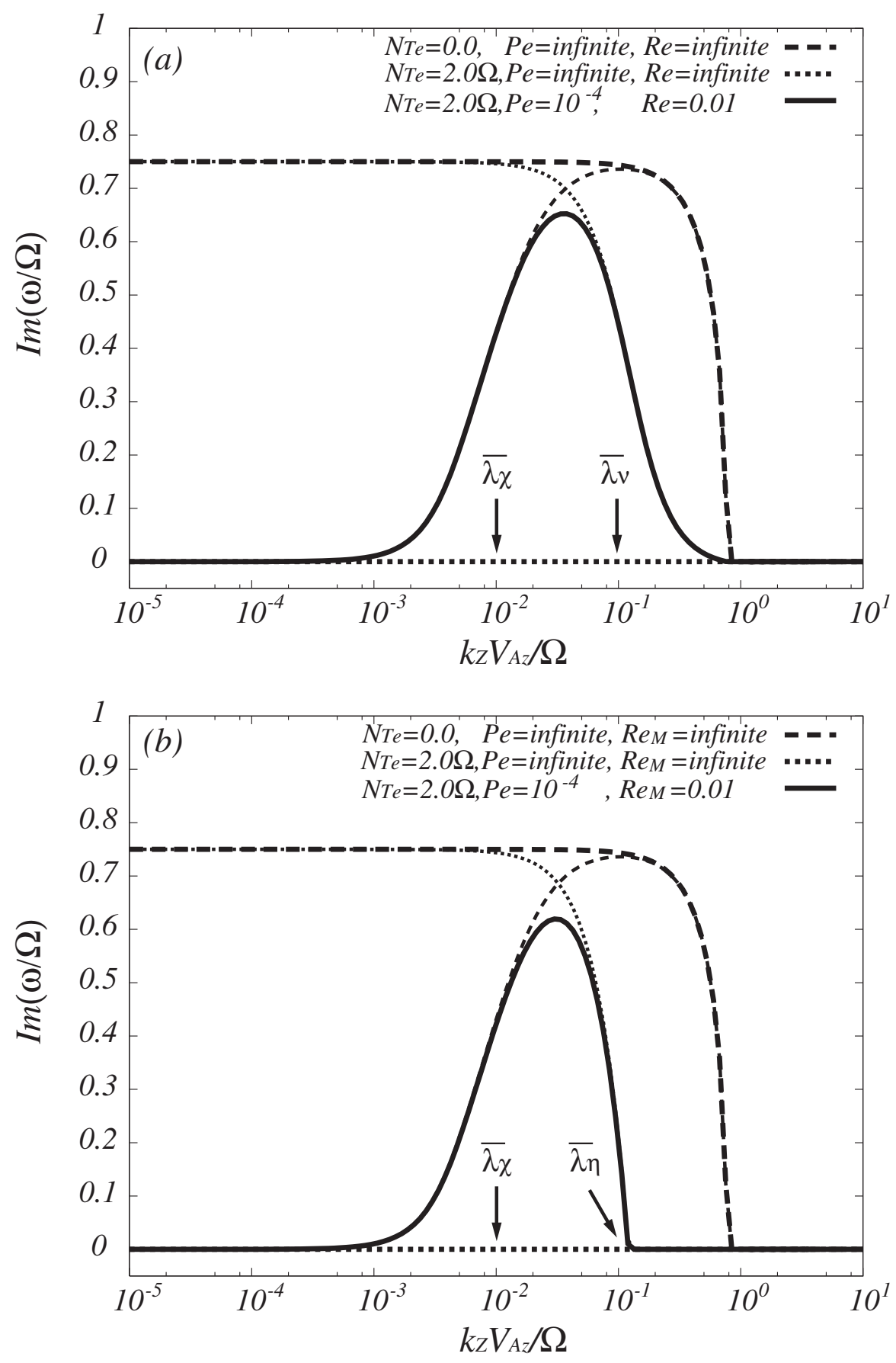

Fig. 4.- Double diffusive effects in (a) the PNS-type systems and (b) the SOLAR-type systems. In the PNS-type systems, the heat and viscous diffusivities are sufficiently larger than the magnetic diffusivity $(\chi \gg \nu \gg \eta)$. On the other hand, in the SOLAR-type systems, the heat and magnetic diffusivities are dominant over the viscous diffusivity $(\chi \gg \eta \gg \nu)$. Unstable growth rates are shown as a function of the normalized vertical wavenumber $k_{z} V_{A z} / \Omega$. Model parameters are the same as those in Figure 3. 

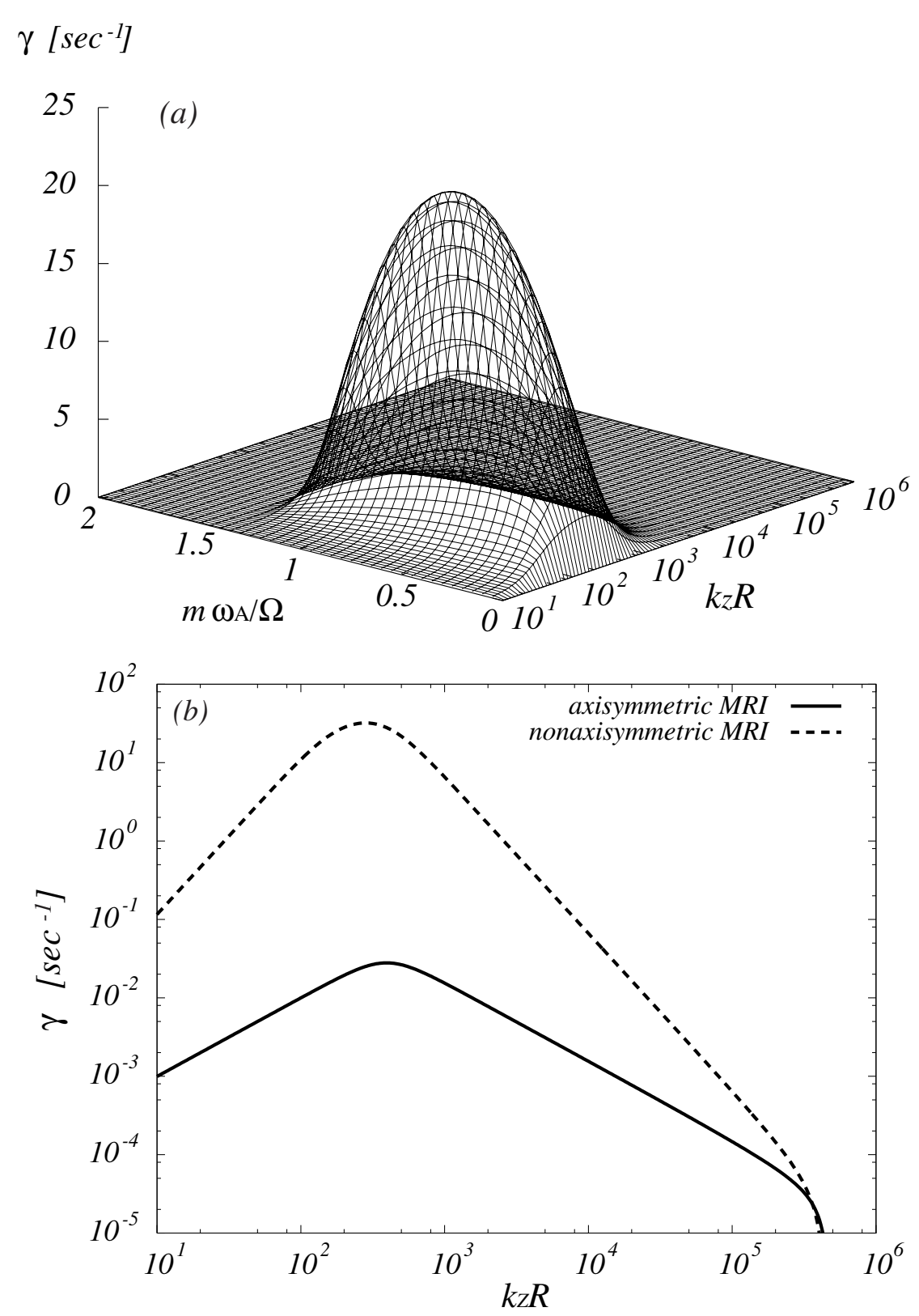

Fig. 5.- (a) Three-dimensional plot of the unstable growth rate as a function of the azimuthal wavenumber $m$ and the normalized vertical wavenumber $k_{z} R$, and (b) slices of the three-dimensional plot at $m=0$ and $m=m_{\max }$. The size of the system is assumed to be $R=3 \times 10^{6} \mathrm{~cm}$. The modes with $m=0$ are corresponding to the axisymmetric MRI (solid line), and the other is the nonaxisymmetric MRI (dashedline). We choose the model parameters as $q=1.0, \Omega=100 \mathrm{sec}^{-1}, N_{T}=N_{L}=10 \Omega \mathrm{sec}^{-1}, \omega_{A}=1.0 \mathrm{sec}^{-1}$, and $\omega_{A z}=10^{-4} \mathrm{sec}^{-1}$. Quadruple diffusivities are assumed as $\chi=10^{12} \mathrm{~cm}^{2} \mathrm{sec}^{-1}, \xi=3 \times 10^{11} \mathrm{~cm}^{2} \mathrm{sec}^{-1}$, $\nu=10^{10} \mathrm{~cm}^{2} \mathrm{sec}^{-1}$, and $\eta=10^{-3} \mathrm{~cm}^{2} \mathrm{sec}^{-1}$, respectively. These figures represent the characteristics of MRI at the equatorial plane of the PNSs. 

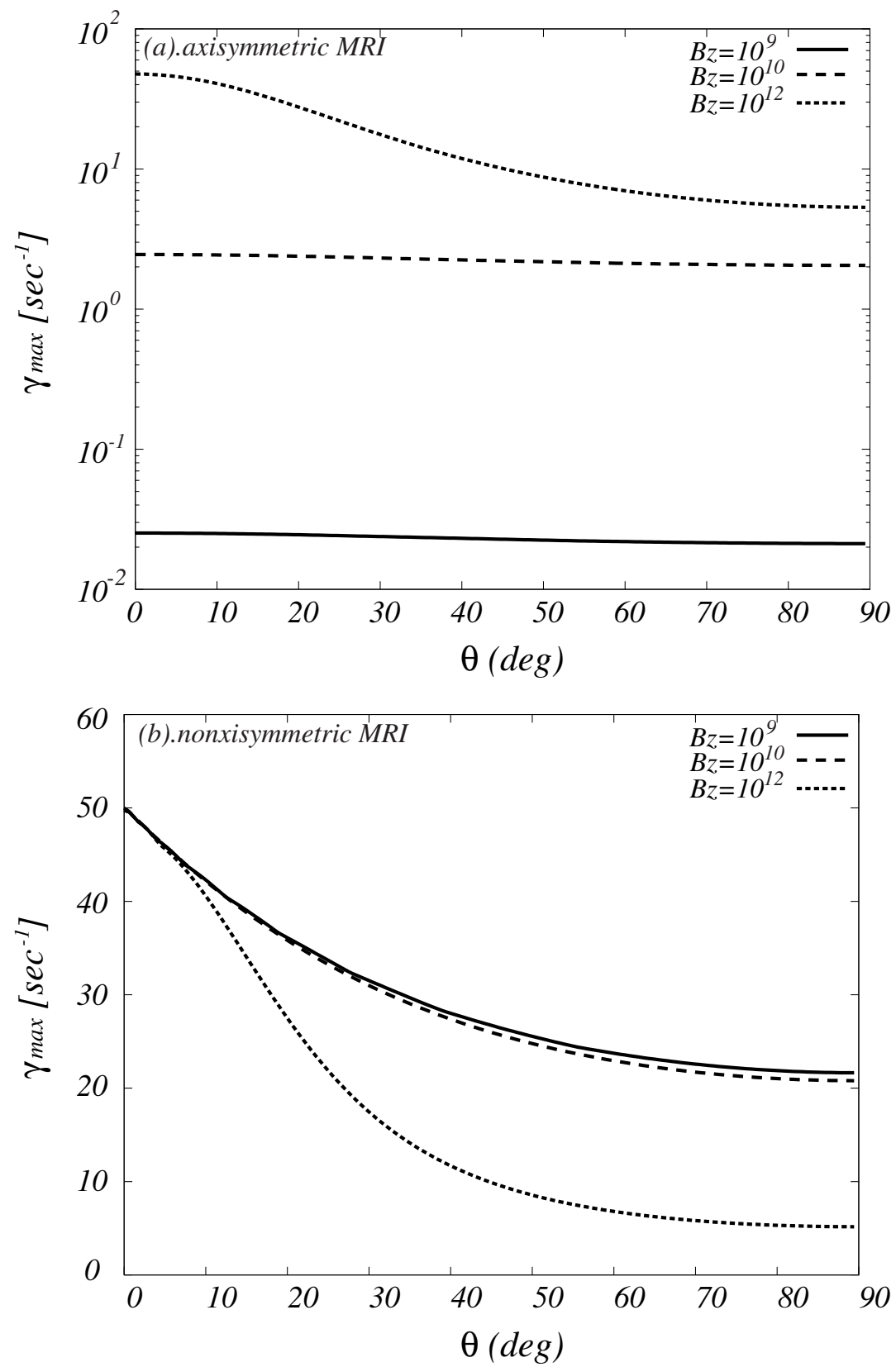

Fig. 6. - The maximum growth rate of the MRI as a function of the polar angle $\theta$ for the cases of $B_{z}=$ $10^{9}, 10^{10}$, and $10^{12} \mathrm{G}$. The upper panel shows the behavior of axisymmetric MRI $(m=0)$, and the lower panel is nonaxisymmetric MRI $(m \neq 0)$. We search the fastest growing modes solving our simplyfied dispersion equation (31). The strength of toroidal fields is assumed to be constant in all cases, $B_{\phi}=10^{13} \mathrm{G}$. The other parameters are the same as those in Figure 5. Note that the vertical axis of upper figure is measured by the logarithmic scale, but that of bottom figure is measured by the normal scale. 